Article

\title{
Nanoporous Crystalline Composite Aerogels with Reduced Graphene Oxide
}

\author{
Christophe Daniel *D, Baku Nagendra, Maria Rosaria Acocella $\mathbb{D}$, Esther Cascone $\mathbb{D}$ \\ and Gaetano Guerra
}

Dipartimento di Chimica e Biologia "Adolfo Zambelli", Università degli Studi di Salerno, Via Giovanni Paolo II 132, 84084 Fisciano, Italy; nbaku@unisa.it (B.N.); macocella@unisa.it (M.R.A.); esthercascone@hotmail.it (E.C.); gguerra@unisa.it (G.G.)

* Correspondence: cdaniel@unisa.it

Academic Editors: Stefano Cardea, Antonio Tabernero de Paz and Silvio A.B. Vieira de Melo Received: 20 October 2020; Accepted: 6 November 2020; Published: 10 November 2020

\begin{abstract}
High-porosity monolithic composite aerogels of syndiotactic polystyrene (sPS) and poly(2,6-dimethyl-1,4-phenylene oxide) (PPO) containing reduced graphene oxide (r-GO) were prepared and characterized. The composite aerogels obtained by supercritical carbon dioxide $\left(\mathrm{scCO}_{2}\right)$ extraction of sPS/r-GO and PPO/r-GO gels were characterized by a fibrillar morphology, which ensured good handling properties. The polymer nanoporous crystalline phases obtained within the aerogels led to high surface areas with values up to $440 \mathrm{~m}^{2} \mathrm{~g}^{-1}$. The role of r-GO in aerogels was studied in terms of catalytic activity by exploring the oxidation capacity of composite PPO and sPS aerogels toward benzyl alcohol in diluted aqueous solutions. The results showed that, unlike sPS/r-GO aerogels, PPO/r-GO aerogels were capable of absorbing benzyl alcohol from the diluted solutions, and that oxidation of c.a. $50 \%$ of the sorbed benzyl alcohol molecules into benzoic acid occurred.
\end{abstract}

Keywords: aerogels; sol-gel process; supercritical carbon dioxide extraction; syndiotactic polystyrene; poly(2,6-dimethyl-1,4-phenylene oxide); reduced graphene oxide; catalytic oxidation

\section{Introduction}

Since the first examples of synthetic polymer aerogels reported by Pekala and co-workers [1-3], many types of polymer aerogels have been synthetized by changing the sol-gel process and the polymer backbone [4-12]. Due to the combination of low cost, simple production processes, and straight-forward tailoring of the physicochemical and functional properties [13-15], polymer aerogels continue to attract significant attention from academic and industrial communities for a diverse range of uses within the scope of a broad technological platform [16-24].

In particular, syndiotactic polystyrene (sPS) and poly(2,6-dimethyl-1,4-phenylene oxide) (PPO) aerogels endow mixed porosity, namely, nanoporosity (or ultra-microporosity according to IUPAC classification; $<0.7 \mathrm{~nm}$ ) located in the polymer crystalline phase as well as mesoporosity and macroporosity within the three-dimensional (3D) fibrillar surface voids [25-27]. The nanoporous crystalline phases of sPS and PPO are able to sorb volatile organic compounds (VOCs) from water and vapour phase even at low activities [28-33]. Nanoporous PPO and sPS aerogels present the high sorption capacity typical of their respective nanoporous crystalline phases associated with a fast sorption kinetics due to high mesoporosity and macroporosity [25-27]. Therefore, sPS and PPO nanoporous crystalline aerogels are particularly interesting for potential use in air and water purification devices. In addition to this possible use, applications in microelectronics [34] and for the removal of airborne nanoparticles $[35,36]$ have been also proposed for sPS aerogels. 
High-porosity polymeric aerogels are also especially appealing for supporting catalysts as they are chemically inert and mechanically stable with high durability and they offer many benefits, such as high surface area and high sorption. Many examples of aerogel catalysts have been reported in literature [37-41].

Graphene oxide (GO) and other carbon-based oxides have long been highly attractive for use as catalysts, catalyst carriers, and catalytic reagents in organic molecule transformations [42-49] due to their unique characteristics, which include enriched chemical structures, functional active sites (acidic and basic), wreckages, defects, unpaired $\pi$-electrons of carbon, and larger specific surface areas. Moreover, the addition of carbon-based materials has been also shown to improve the efficiency of metal oxide photocatalysts [50,51]. As the catalytic efficiency strongly depends on active sites and available surface area of the catalyst [43-45,49], undesired large polydisperse particles are formed. The prevention of aggregation is also essential to fully exploit the physical properties of GO and obtain composite materials with superior functional properties [50,52-54].

In this study, we fabricated monolithic aerogels based on nanoporous crystalline PPO and sPS polymers and reduced graphene oxide (r-GO) hybrid nanocomposites by making use of the advantages of nanoporous crystallinity of the polymers as well as the GO catalytic activity. The uniform dispersion of r-GO was controlled in a two-step approach, gelation followed by supercritical carbon dioxide $\left(\mathrm{scCO}_{2}\right)$ extraction procedure. The monolithic composite sPS/r-GO and PPO/r-GO aerogels were characterized by a fibrillar morphology, which ensured good mechanical properties, and large surface area values up to $440 \mathrm{~m}^{2} \mathrm{~g}^{-1}$ for PPO/r-GO aerogels.

The oxidizing ability of composite aerogels and the possible use of these materials as selective heterogeneous catalytic agents for the transformations of organic pollutants from aqueous solutions were assessed by exploring the oxidation of benzyl alcohol from diluted aqueous solutions, as GO displays a high propensity to oxidize alcohols. Preliminary tests reported here showed that, while sPS/r-GO aerogels did not present any oxidation of benzyl alcohol as a result of the absence of sorption within the aerogels, PPO/r-GO aerogels were capable of absorbing benzyl alcohol from the diluted aqueous solutions and oxidizing a fraction of the sorbed benzyl alcohol molecules.

\section{Results}

\subsection{Reduced Graphite Oxide (r-GO) Preparation and Characterization}

The X-ray diffraction patterns of the starting graphite $(\mathrm{G})$, the resultant graphene oxide (GO) obtained by the Hummers' method, and the final reduced graphene oxide (r-GO) obtained by solvothermal reduction followed by extraction with $\mathrm{scCO}_{2}$ are reported in Figure 1.

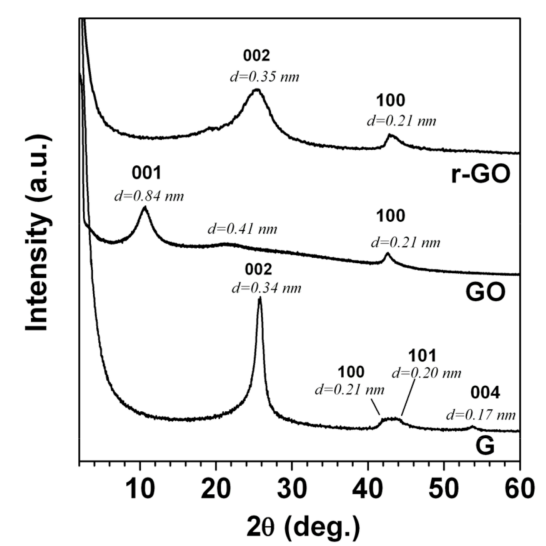

Figure 1. X-ray diffraction patterns of graphite $(\mathrm{G})$, the resultant graphene oxide (GO) obtained by the Hummers' method, and the final reduced graphene oxide (r-GO) obtained by solvothermal reduction. 
The oxidized graphite (GO) formation was confirmed by an increase in the distance between the layers from $0.34 \mathrm{~nm}$ up to $0.84 \mathrm{~nm}$, while the $d$-spacing corresponding to the in-plane periodicities of 100 planes remained intact. After solvothermal reduction of GO, we observed the X-ray diffraction pattern, the maintenance of the in-layer graphitic order (100 reflection plans), and the displacement of the peak ( $d=0.84 \mathrm{~nm}$ with a peak at $d=0.35 \mathrm{~nm}$ ), which clearly indicated the formation of r-GO. Another notable difference was in the full-width at half maxima (FWHM) of the r-GO, which increased compared with that of pristine graphite $(\mathrm{G})$, confirming the formation of the disordered graphitic structure after oxidation followed by solvothermal reduction treatments.

\subsection{Polymer/r-GO Monolithic Aerogel Preparation and Characterization}

Schematic illustration (Figure 2A) shows the method for the preparation of homogeneously dispersed monolithic aerogels of polymer/r-GO nanocomposites.

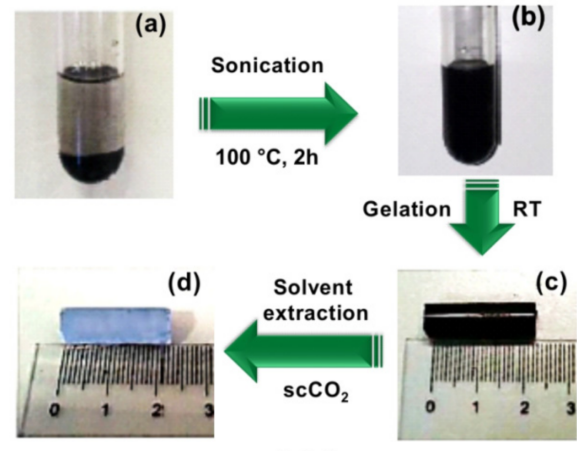

(A)

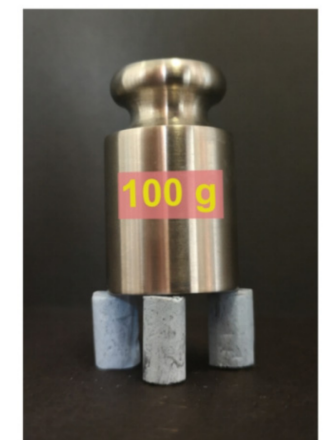

(B)

Figure 2. (A) Preparation procedure for monolithic polymer/r-GO aerogel: dispersion of GO into an organic solvent before (a) and after (b) sonication at $100{ }^{\circ} \mathrm{C}$ for $2 \mathrm{~h}$ in step one; (c) stable self-standing gel formed after adding the polymer to the dispersion of GO and the dissolution of the polymer at high temperature; (d) monolithic aerogel of polymer/r-GO nanocomposites fabricated by supercritical carbon dioxide $\left(\mathrm{scCO}_{2}\right)$ extraction of gels obtained in step two. (B) Three syndiotactic polystyrene $(\mathrm{sPS}) / \mathrm{r}-\mathrm{GO}$ aerogel pillars (porosity $=90 \%$ ) supporting $100 \mathrm{~g}$.

In the first step, the freshly prepared GO was dispersed in 1,4-dichlorobenzene (DCB) solvent (see photo a). Subsequently, solvothermal reduction treatment was performed in a bath sonicator, which promoted the simultaneous reduction of oxygenated functional groups such as epoxy, hydroxyl, and carboxylic groups, as well as the removal of surface $\mathrm{H}_{2} \mathrm{O}$ molecules from the GO structure, leading to the weakening of the interlayer attraction. On further sonication, complete layer separation took place, resulting in an exfoliated and stable dispersion in the hydrophobic solvent (see photo b). Then, polymer was added to this stable dispersion and after complete dissolution of the polymer and obtaining a homogeneous solution by heating, the solution was cooled down to room temperature where the gelation occurred (see photo c). Subsequently, the gel solvent was extracted with supercritical carbon dioxide $\left(\mathrm{scCO}_{2}\right)$, which led to the formation of monolithic aerogels with uniformly dispersed polymer/r-GO nanocomposites, as shown in the final step (see photo d).

$\mathrm{ScCO}_{2}$ solvent extraction procedure offered a monolithic aerogel of hybrid polymer/r-GO nanocomposites with retention of both the shape and size of the gel with negligible shrinkage (Figure 2A; photos a and $\mathrm{b}$ ). It is also worth noting that the monolithic composite aerogels displayed good handling properties as can be seen in Figure 2B.

For monolithic aerogels with a regular shape, the total porosity, including macroporosity, mesoporosity, and microporosity, can be estimated from the volume/mass ratio of the aerogel. The density and total porosity values of neat polymer aerogels and polymer/r-GO aerogels obtained with sPS and PPO are listed in Table 1. 
Table 1. Summary of the apparent density $\left(\mathrm{g} \mathrm{cm}^{-3}\right)$, total porosity $\left(\mathrm{cm}^{3} \mathrm{~g}^{-1}\right)$, pore volume $\left(\mathrm{cm}^{3} \mathrm{~g}^{-1}\right)$, mesopore radius $(\mathrm{nm})$, and surface area $\left(\mathrm{S}_{\mathrm{BET}}, \mathrm{m}^{2} \mathrm{~g}^{-1}\right)$ of the pure polymer aerogels and hybrid polymer aerogels with $10 \mathrm{wt}$.\% polymer of the total solvent weight and 80/20 polymer/r-GO weight fraction.

\begin{tabular}{ccccc}
\hline & sPS & sPS $_{\mathbf{0 . 8}} / \mathbf{r}-\mathbf{G O}_{0.2}$ & PPO & PPO $_{\mathbf{0 . 8}} / \mathbf{r}-\mathbf{G O}_{\mathbf{0 . 2}}$ \\
\hline Apparent density $\left(\mathrm{g} \mathrm{cm}^{-3}\right)$ & 0.15 & 0.16 & 0.18 & 0.20 \\
Total porosity ${ }^{\mathrm{a}}\left(\mathrm{cm}^{3} \mathrm{~g}^{-1} / \%\right)$ & $6.66 / 86$ & $6.25 / 80$ & $5.55 / 82$ & $5 / 76$ \\
$\mathrm{~S}_{\mathrm{BET}} \mathrm{b}^{2}\left(\mathrm{~m}^{2} / \mathrm{g}\right)$ & $290 \pm 20$ & $250 \pm 20$ & $550 \pm 10$ & $440 \pm 10$ \\
$\mathrm{~V}_{\text {tot }} \mathrm{N}_{2}{ }^{\mathrm{c}}\left(\mathrm{cm}^{3} \mathrm{~g}^{-1}\right)$ & 0.56 & 0.41 & 1.013 & 0.628 \\
$\mathrm{~V}_{\text {meso }}{ }^{\mathrm{d}}\left(\mathrm{cm}^{3} \mathrm{~g}^{-1}\right)$ & 0.49 & 0.33 & 0.86 & 0.48 \\
Average mesopore radius $\mathrm{d}(\mathrm{nm})$ & 9.0 & 6.3 & 8.9 & 8.7 \\
$\mathrm{~V}_{\text {micro }}{ }^{\mathrm{e}}\left(\mathrm{cm}^{3} \mathrm{~g}^{-1}\right)$ & 0.049 & 0.046 & 0.13 & 0.12 \\
\hline
\end{tabular}

${ }^{a}$ Total porosity estimated from the volume/mass ratio expressed as $\mathrm{cm}^{3} \cdot \mathrm{g}^{-1}$ and $\%$ using Equation (1). ${ }^{\mathrm{b}}$ Total area evaluated following the BET model. ${ }^{c}$ Pore volume $\left(\mathrm{V}_{\text {tot }} \mathrm{N}_{2}\right)$ calculated as the volume of $\mathrm{N}_{2}$ liquid at $\mathrm{P} / \mathrm{P}_{0} \approx 0.95$. ${ }^{\mathrm{d}}$ Average mesopore radius and $\mathrm{V}_{\text {meso }}$ calculated with the $\mathrm{BJH}$ method. ${ }^{\mathrm{e}}$ Cumulative micropore volume obtained from DFT analysis. PPO, poly(2,6-dimethyl-1,4-phenylene oxide).

The typical SEM pictures of a pure sPS aerogel and a hybrid sPS/r-GO aerogel are compared in Figure 3.

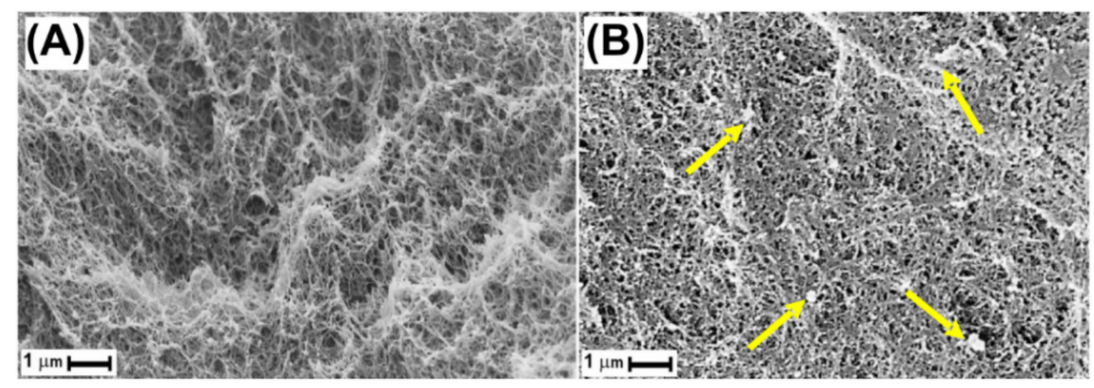

Figure 3. SEM images of the morphology of the monolithic pristine sPS aerogel (A) and of the hybrid sPS/r-GO aerogel obtained after $\mathrm{scCO}_{2}$ solvent extraction (B). Some r-GO particles dispersed in the hybrid aerogel are indicated by yellow arrows.

The SEM pictures (Figure 3A,B) showed that, both for pristine sPS aerogel and the hybrid sPS/r-GO aerogel, an open network of fibrils with a diameter of 50-200 nm was obtained. Most pores of the network were $0.1-0.5 \mu \mathrm{m}$ wide. We were also able to observe, on the SEM micrograph of the composite aerogel (Figure 3B), the absence of large size r-GO aggregates. In fact, the SEM images displayed the presence of r-GO particles (some of them are indicated by yellow arrows) dispersed in the monolithic polymer aerogels having an average lateral size of c.a. $200-750 \mathrm{~nm}$. The same fibrillar morphology was also observed for PPO-based aerogels (not shown here).

Figure 4 shows the $\mathrm{X}$-ray diffraction patterns of r-GO and hybrid polymer/r-GO nanocomposite monolithic aerogels containing $20 \mathrm{wt} . \%$ of r-GO. 


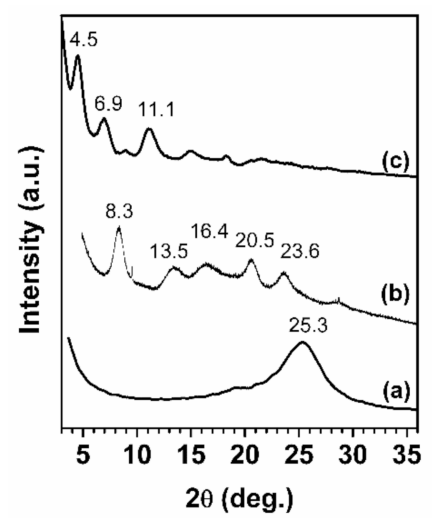

Figure 4. X-ray diffraction patterns of r-GO (a) and hybrid monolithic polymer aerogels of $\mathrm{sPS}_{0.8} / \mathrm{r}-\mathrm{GO}_{0.2}(\mathrm{~b})$, and $\mathrm{PPO}_{0.8} / \mathrm{r}-\mathrm{GO}_{0.2}$ (c) nanocomposites containing $20 \mathrm{wt} . \%$ of $\mathrm{r}-\mathrm{GO}$.

The 002 reflection corresponding to r-GO at $2 \theta=25.3^{\circ}$ was not detected in any of the two nanocomposite aerogels. This absence of the r-GO reflection clearly indicated that most of the GO was constituted by structural layers exhibiting negligible order in the direction perpendicular to the graphitic structure due to homogeneous dispersion of r-GO nanoparticles in the polymer matrix [55].

The X-ray diffraction patterns also showed that, after the guest (solvent) extraction procedure, nanoporous crystalline polymer phases were obtained for both composite aerogels. Curve b of Figure 4 showed diffraction reflections at $2 \theta=8.3^{\circ}, 13.5^{\circ}, 16.4^{\circ}, 20.5^{\circ}$, and $23.6^{\circ}$, typical of the known crystalline nanoporous structure, $\delta$-phase [56], for the $\mathrm{sPS}_{0.8} / \mathrm{r}-\mathrm{GO}_{0.2}$ monolithic aerogel, while the $\mathrm{PPO}_{0.8} / \mathrm{r}-\mathrm{GO}_{0.2}$ aerogel diffraction pattern (curve $\mathrm{c}$ of Figure 4) appeared with diffraction reflections at $2 \theta=4.5^{\circ}, 6.9^{\circ}$, and $11.1^{\circ}$ corresponding to the $\alpha$ PPO nanoporous crystalline structure modification [57].

Sorption-desorption $\mathrm{N}_{2}$ isotherms (where sorption is expressed as $\mathrm{cm}^{3}$ of nitrogen in normal conditions per gram of polymer) collected for pristine polymer aerogels and composite aerogels containing $20 \mathrm{wt.} \%$ of r-GO are reported in Figure 5.

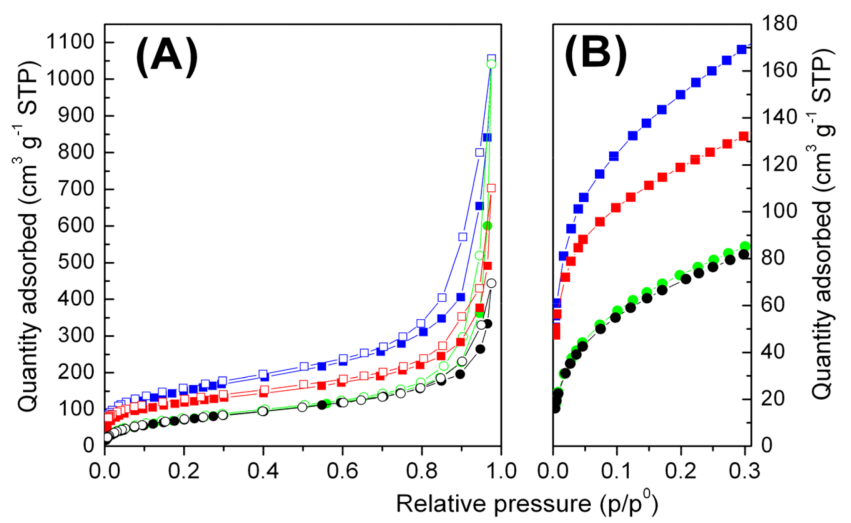

Figure 5. Volumetric $\mathrm{N}_{2}$ adsorption-desorption isotherms at $77 \mathrm{~K}$ in the $0-1 \mathrm{P} / \mathrm{P}_{0}$ range (A) and in the $0-0.3 \mathrm{P} / \mathrm{P}_{0}$ range $(\mathrm{B})$ of pure sPS (circles, green) and $\mathrm{PPO}$ (squares, blue) aerogels and of sPS/r-GO (circles, black) and PPO/r-GO (squares, red) nanocomposites aerogels containing $20 \mathrm{wt}$. $\%$ of r-GO. Filled and empty scatters refer to the adsorption and desorption branches, respectively.

The shape of isotherms, associated with the presence of hysteresis, suggested type IV isotherm with the presence of big mesopores.

The $\mathrm{N}_{2}$ physisorption at $77 \mathrm{~K}$ allows to characterize pores in the mesopore and micropore range. The total meso and macropore volume can be customarily estimated from the volume of the liquid $\mathrm{N}_{2}$ sorbed at a predetermined $\mathrm{P} / \mathrm{P}_{0}$ value in the upper part of the isotherm curve. However, the presence of macropores shown by the SEM measurements (Figure 3) associated with large mesopores precluded an 
accurate determination of the total pore volume since adsorbed gas volumes increased asymptotically as relative pressure approached unity. The total pore volume $\left(\mathrm{V}_{\text {tot }} \mathrm{N}_{2}\right)$ estimated as the volume of liquid $\mathrm{N}_{2}$ at $\mathrm{P} / \mathrm{P}_{0} \approx 0.95$ (row 5 of Table 1 ) differed greatly from the total porosity (i.e., macro-, meso-, and micro-porosity) of the aerogels (row 3 of Table 1). We also noted that the mesoporosity calculated with the BJH method (row 6 of Table 1) represented only a small fraction of the total porosity. This result agreed with those of the SEM micrographs (Figure 3) that showed the strong macroporous nature of all the aerogels.

In addition to the $\mathrm{BJH}$ analysis which revealed an average mesopore radius of c.a. $9 \mathrm{~nm}$ for all the aerogels with the exception of the $\mathrm{sPS}_{0.8} / \mathrm{r}-\mathrm{GO}_{0.2}$ aerogel which displayed an average radius of $6.3 \mathrm{~nm}$ (row 7 of Table 1), the presence of microporosity was also verified by the DFT method. The DFT analysis showed that micropores were present in the range of $0.13-0.20 \mathrm{~nm}$ and it also appeared that the micropore volumes were about two times larger for PPO aerogels than the values calculated for sPS aerogels (row 8 of Table 1). These larger values can be attributed to the nanoporous nature of both the amorphous and the crystalline phases of PPO samples obtained after solvent removal [30], while for sPS, only the crystalline phase was nanoporous.

The DFT results were consistent with the BET values (row 4 of Table 1). For instance, the measured surface area for the pure PPO aerogel was $550 \mathrm{~m}^{2} \mathrm{~g}^{-1}$, while the BET value obtained for the pure sPS aerogel was $290 \mathrm{~m}^{2} \mathrm{~g}^{-1}$. For both PPO and sPS nanocomposite aerogels, a decrease in the surface area was observed. In first approximation, this decrease could be attributed to the r-GO fraction as the r-GO powder used for the preparation of the composite aerogels exhibited a value of $2 \mathrm{~m}^{2} \mathrm{~g}^{-1}$.

It is worth noting that even though the amount of r-GO in the composite aerogel was $20 \mathrm{wt} . \%$, the BET value of $\mathrm{PPO}_{0.8} / \mathrm{r}-\mathrm{GO}_{0.2}$ was as high as $440 \mathrm{~m}^{2} \mathrm{~g}^{-1}$. This feature is essential, as catalyst supports must provide a considerably large surface area.

\subsection{Catalytic Activity of the Polymer/r-GO Monolithic Aerogels}

In order to assess the catalytic activity of sPS/r-GO and PPO/r-GO aerogels and the possible use of these composite aerogels for the degradation of organic compounds from water, benzyl alcohol was selected as a model compound for preliminary testing. The oxidation scheme of benzyl alcohol by GO reported in literature [42] is shown in Figure 6.

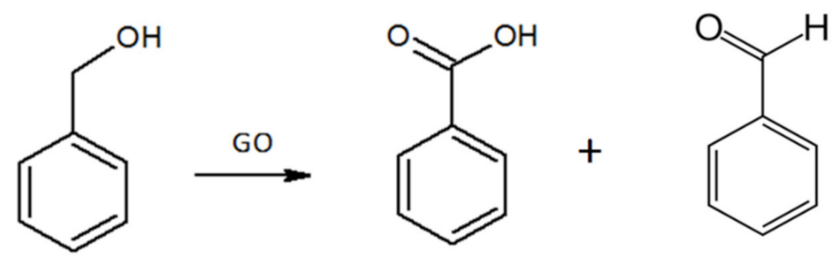

Figure 6. Reaction scheme shows benzyl alcohol oxidation in the presence of GO catalyst.

The oxidation of benzyl alcohol in the presence of GO as catalyst leads to the formation of benzaldehyde and benzoic acid, which are obtained in different relative amounts depending on the amount of GO and the reaction conditions [42].

In Figure 7 , the FTIR spectra of $\mathrm{sPS}_{0.8} / \mathrm{r}_{-}-\mathrm{GO}_{0.2}$ aerogels before (curve ii) and after (curve iii) immersion in a $100 \mathrm{ppm}$ benzyl alcohol aqueous solution are reported. For the sake of discussion, the FTIR spectrum of benzyl alcohol is also reported (curve i). 


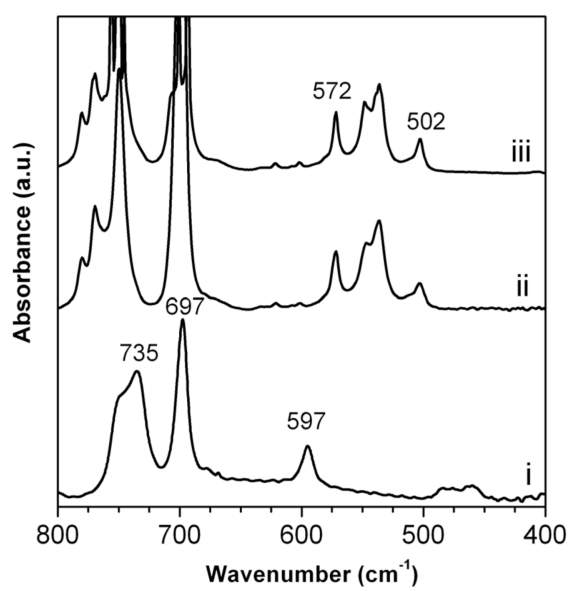

Figure 7. FTIR spectra of liquid benzyl alcohol (curve i) and sPS ${ }_{0.8} / \mathrm{r}-\mathrm{GO}_{0.2}$ aerogel $(\mathrm{P}=90 \%$ ) before (curve ii) and after (curve iii) immersion for 3 days in a 100 ppm benzyl alcohol aqueous solution.

The FTIR spectra of the sPS/r-GO aerogel before (curve ii) and after (curve iii) immersion in a $100 \mathrm{ppm}$ benzyl alcohol aqueous solution presented IR bands located at 572 and $502 \mathrm{~cm}^{-1}$ typical of the ordered $\mathrm{s}(2 / 1) 2$ helical conformation [58], which is characteristic of the nanoporous crystalline $\delta$-form. From Figure 7, we could also observe that the FTIR spectrum of the aerogel before (curve i) and after (curve ii) immersion in the aqueous solution of benzyl alcohol remained unchanged. In particular, none of the benzyl alcohol IR bands at 735 and $597 \mathrm{~cm}^{-1}$ (see curve i), which had not covered by sPS bands, were observed on the FTIR spectrum collected after immersion. This result indicated that benzyl alcohol was not absorbed from the aqueous solution by the aerogel.

In Figure 8A,B, the FTIR spectra of PPO aerogels and PPO/r-GO aerogels before (curves i) and after (curves ii) immersion in a 100 ppm benzyl alcohol solution are reported.
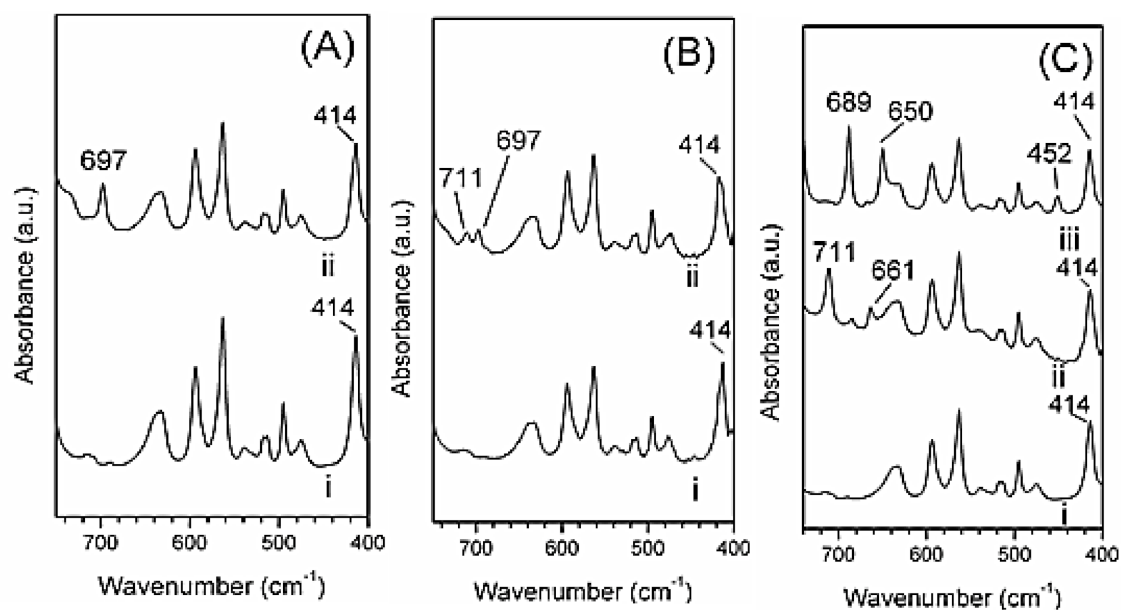

Figure 8. (A) FTIR spectra of PPO aerogels before (curve i) and after (curve ii) immersion in a $100 \mathrm{ppm}$ aqueous solution of benzyl alcohol; (B) FTIR spectra of $\mathrm{PPO}_{0.8} / \mathrm{r}-\mathrm{GO}_{0.2}$ aerogels before (curve i) and after (curve ii) immersion for 4 days in a 100 ppm benzyl alcohol solution; (C) FTIR spectra of PPO aerogels before (curve i) and after immersion in a 100 ppm solution of benzoic acid (curve ii) and benzaldehyde (curve iii).

First of all, it is worth noting that all the FTIR spectra of both pure PPO and PPO/r-GO aerogels displayed a band located at $414 \mathrm{~cm}^{-1}$, which is typical of the PPO nanoporous crystalline phase [30]. The FTIR spectrum of the PPO aerogel collected after immersion in the aqueous benzyl alcohol solution (curve ii) presented a new band at $697 \mathrm{~cm}^{-1}$ (Figure 8A). The presence of this typical band of benzyl 
alcohol (see curve i of Figure 7) showed that benzyl alcohol was sorbed by the aerogel. The amount of benzyl alcohol absorbed from the aqueous solution determined by TGA was c.a. $7 \mathrm{wt} . \%$. The ability of PPO to sorb benzyl alcohol, while it was not absorbed by sPS aerogels, could be attributed to the different shape and size of the PPO crystalline nanopores [27].

The FTIR spectrum of the composite $\mathrm{PPO}_{0.8} / \mathrm{r}_{-} \mathrm{GO}_{0.2}$ aerogel collected after immersion in the solution (Figure 8B) differed from the spectrum obtained with the neat PPO aerogel. In particular, with respect to curve ii of Figure 8A, a decrease in the benzyl alcohol characteristic band at $697 \mathrm{~cm}^{-1}$ and the appearance of a new band at $711 \mathrm{~cm}^{-1}$ (curve ii of Figure $8 \mathrm{~B}$ ) were observed. This result might indicate a partial conversion of benzyl alcohol.

As mentioned above (Figure 6), the oxidation of benzyl alcohol by r-GO can lead to the formation of benzaldehyde and benzoic acid. Thus, in order to determine which compound was formed in the composite PPO/r-GO aerogel, the FTIR spectra of neat PPO aerogel after sorption of benzaldehyde and benzoic acid from $100 \mathrm{ppm}$ aqueous solutions were collected (Figure 8C).

After sorption of benzaldehyde, the FTIR spectrum of the PPO aerogel displayed strong absorbance bands located at 689 and $650 \mathrm{~cm}^{-1}$ (curve iii of Figure 8C), while the appearance of a strong band located at $711 \mathrm{~cm}^{-1}$ (curve ii of Figure $8 \mathrm{C}$ ) was observed after sorption of benzoic acid. This result suggested that the oxidation of sorbed benzyl alcohol in the PPO/r-GO aerogel led to the formation of benzoic acid. Based on the decrease in the absorbance of the $697 \mathrm{~cm}^{-1}$ band typical of benzyl alcohol, the fraction of benzyl alcohol being oxidized could be estimated as c.a. $50 \%$.

\section{Materials and Methods}

\subsection{Materials}

The high molecular weight PPO (Ultra High P6130 grade; $\mathrm{M}_{\mathrm{w}}=350 \mathrm{~kg} \mathrm{~mol}^{-1}$ ) and sPS (90ZC grade; $\mathrm{M}_{\mathrm{w}}=200 \mathrm{~kg} \mathrm{~mol}^{-1}$ ) used in this study were kindly supplied by Sabic (Bergen op Zoom, The Netherlands) and Idemitsu Kosan Co., Ltd. (Tokyo, Japan), respectively. Graphite, with an 8427 trademark, was purchased from Asbury Graphite Mills Inc. (Asbury, OH, USA) Solvents and other reagents used in this work were purchased from Sigma Aldrich (St. Louis, MO, USA) and used as received.

\subsection{Graphene Oxide and Reduced Graphene Oxide Synthesis}

Hummers' method was adapted to the synthesis of graphite oxide (GO) [59]. Briefly, $120 \mathrm{~mL}$ of $\mathrm{H}_{2} \mathrm{SO}_{4}$ and $2.5 \mathrm{~g}$ of $\mathrm{NaNO}_{3}$ were introduced into a $2000 \mathrm{~mL}$ three-neck round-bottomed flask containing $5 \mathrm{~g}$ of pure graphite powder. After that, $15 \mathrm{~g}$ of $\mathrm{KMnO}_{4}$ was also added slowly with magnetic stirring; during this process, the oil bath temperature was maintained below $10^{\circ} \mathrm{C}$. Subsequently, the reaction temperature was increased to $35^{\circ} \mathrm{C}$ and continued overnight. At the end of the reaction, $700 \mathrm{~mL}$ of deionized water and $5 \mathrm{~mL}$ of $\mathrm{H}_{2} \mathrm{O}_{2}(30 \mathrm{wt} . \%)$ was added to the reaction mixture and removed from the oil bath. Additionally, $7 \mathrm{~L}$ of deionized water was added and then centrifuged at 10,000 rpm for $15 \mathrm{~min}$ with a Hermle Z $323 \mathrm{~K}$ centrifuge. Then, the isolated GO powders were repeatedly washed with a $5 \mathrm{wt} . \% \mathrm{HCl}$ aqueous solution and later with deionized water. Finally, the GO powders were collected and dried at $60^{\circ} \mathrm{C}$ for $12 \mathrm{~h}$.

The r-GO dispersions were obtained by solvothermal reduction treatment by the addition of the appropriate amount of GO in $5 \mathrm{~mL}$ of $O$-dichlorobenzene, followed by sonication treatment in a $5000 \mathrm{~mL}$ batch bath sonicator (Badelin Sonorex RK $1028 \mathrm{H}$ ) at $100{ }^{\circ} \mathrm{C}$ for $120 \mathrm{~min}$. Then, powder was recovered by filtration followed by $\mathrm{O}$-dichlorobenzene extraction with $\mathrm{scCO}_{2}$.

\subsection{Monolithic Polymer Aerogels Fabrication}

Two different solvent systems were used in gel preparation with respect to the polymer type. For example, sPS polymer gels were prepared with O-dichlorobenzene, whereas the PPO polymer gels were prepared with $\mathrm{CCl}_{4}$ solvent. 
Gels were prepared in hermetically sealed test tubes by heating the mixture until complete dissolution of the polymer and obtaining a homogeneous solution. The hot solution was cooled down to room temperature, where gelation occurred. Subsequently, the prepared gels were extracted with a SFX 200 supercritical carbon dioxide extractor (ISCO Inc., Lincoln, NE, USA), using the following conditions: temperature $(\mathrm{T}), 40^{\circ} \mathrm{C}$; pressure $(\mathrm{P}), 250$ bar; extraction time duration, $5 \mathrm{~h}$.

The corresponding polymer/r-GO nanocomposite gels $\left(\mathrm{sPS}_{0.8} / \mathrm{r}-\mathrm{GO}_{0.2}\right.$ and $\left.\mathrm{PPO}_{0.8} / \mathrm{r}-\mathrm{GO}_{0.2}\right)$ with $20 \mathrm{wt} . \%$ of r-GO loading were also prepared. Here, we used the polymer and r-GO, including the concentration as $10 \mathrm{wt} . \%$ of the total solvent weight $\left(C_{\text {pol }}=10 \%\right)$.

The method for polymer/r-GO nanocomposite gel preparation and solvent extraction was the same as that followed for the neat polymer gels, except for one condition. Before complete dissolution of the polymer at high temperature, the GO dispersed solution was initially sonicated for two hours at $100{ }^{\circ} \mathrm{C}$.

\subsection{Characterization}

For samples of monolithic aerogels with a regular cylindrical shape, the total porosity, including macroporosity, mesoporosity, and microporosity, can be estimated from the volume/mass ratio of the aerogel.

Then, the percentage of porosity $(P)$ of the aerogel samples can be expressed as follows [60]:

$$
P=100\left(1-\frac{\rho_{\text {app }}}{\rho_{S}}\right)
$$

where $\rho_{s}$ is the aerogel skeletal density (i.e., polymer matrix or polymer/r-GO matrix) and $\rho_{a p p}$ is the aerogel apparent density calculated from the mass/volume ratio of the monolithic aerogels.

X-ray diffraction measurements were performed with a Bruker D8 automatic diffractometer (Billica, MA, USA). It was operated at a step size of $0.03^{\circ}$, at a rate of $164 \mathrm{~s} / \mathrm{step}$, and with nickel-filtered $\mathrm{Cu} \mathrm{K} \alpha$ radiation. Fourier transform infrared (FTIR) spectra were obtained from the Vertex 70 Bruker spectrometer equipped with a deuterated triglycine sulfate (DTGS) detector and a Ge/KBr beam splitter. The frequency scale was internally calibrated to $0.01 \mathrm{~cm}^{-1}$ using He-Ne laser. All measurements were made at $2.0 \mathrm{~cm}^{-1}$ resolution and 32 scans to reduce the noise level.

The morphology of the monolithic aerogels was characterized by a scanning electron microscope (SEM, Zeiss Evo50 equipped with an Oxford energy dispersive X-ray detector, Oxford, UK), with a low energy electron beam $(5 \mathrm{keV})$. In order to avoid the surface charge and to improve the image resolution, the samples were gold-coated $(\sim 20 \mathrm{~nm})$, by VCR high resolution indirect ion-beam sputtering system, before imaging.

Surface area and pore volumes of monolithic polymer aerogels were obtained by $\mathrm{N}_{2}$ adsorption measurements carried out at $77 \mathrm{~K}$ on a Nova Quantachrome 4200e sorption analyzer (Odelzhausen, Germany). The specific surface area of the aerogels was calculated using the Brunauer-Emmet-Teller method. The mesopore volumes and average radii were calculated by the Barrett-Joyner-Halenda $(\mathrm{BJH})$ method using the desorption branch, while micropore cumulative volume was evaluated using the DFT method.

The sorption and oxidation tests of benzyl alcohol from aqueous solutions with sPS and PPO composite aerogels were conducted using thin pieces of aerogel (thickness, $\sim 0.5 \mathrm{~mm}$; weight, $\sim 5 \mathrm{mg}$ ) soaked in a $200 \mathrm{~mL}$ solution. Benzyl alcohol uptake from diluted aqueous solutions was quantified by thermogravimetric measurements (TGA), using TG 209 F1 from Netzsch Instruments (Selb, Germany). All measurements were performed under controlled nitrogen gas flow and a heating rate of $10^{\circ} \mathrm{C} / \mathrm{min}$.

\section{Conclusions}

SPS/r-GO and PPO/r-GO thermoreversible gels can be easily prepared by dissolution of sPS and $\mathrm{PPO}$ in r-GO dispersions in organic solvents. After the extraction of the solvents from native gels with 
supercritical carbon dioxide, high-porosity monolithic composite aerogels can readily be obtained. These aerogels are characterized by a fibrillar morphology, which ensures good handling properties and a homogeneous dispersion of r-GO within the fiber network.

The X-ray diffraction analysis showed that nanoporous crystalline phases were obtained within the aerogels and this led to the high BET values for the sPS-based aerogels $\left(290 \mathrm{~m}^{2} \mathrm{~g}^{-1}\right)$ and PPO-based aerogels $\left(440 \mathrm{~m}^{2} \mathrm{~g}^{-1}\right)$.

The oxidation tests carried out with dilute aqueous solutions of benzyl alcohol clearly showed that, unlike sPS-based aerogels, PPO-based aerogels were capable of absorbing benzyl alcohol from the dilute solutions and oxidation of c.a. $50 \%$ of the sorbed benzyl alcohol molecules into benzoic acid occurred.

Author Contributions: E.C. carried out the preparation and characterization of r-GO. M.R.A. provided assistance and direction in r-GO preparation. B.N. carried out the preparation and characterization of aerogel samples. C.D. provided assistance and direction in sample preparation and characterization. G.G. provided overall project direction and advice. B.N. and C.D. wrote the manuscript. All authors have read and agreed to the published version of the manuscript.

Funding: This research received no external funding.

Acknowledgments: Nagendra Baku was supported by a grant from Ministero dell'Istruzione, dell’Università e della Ricerca (PON “R\&I” 2014-2020; ARS01_01398 "INPOS-Tecnologia innovativa di rimozione di inquinanti da acqua basati su polimeri cristallini nanoporosi").

Conflicts of Interest: The authors declare no conflict of interest.

\section{References}

1. Pekala, R.W. Organic aerogels from the polycondensation of resorcinol with formaldehyde. J. Mater. Sci. 1989, 24, 3221-3227. [CrossRef]

2. Pekala, R.W.; Alviso, C.T.; Kong, F.M.; Hulsey, S.S. Aerogels derived from multifunctional organic monomers. J. Non-Cryst. Solids 1992, 145, 90-98. [CrossRef]

3. Pekala, R.W.; Alviso, C.T.; Lu, X.; Gross, J.; Fricke, J. New organic aerogels based upon a phenolic-furfural reaction. J. Non-Cryst. Solids 1995, 188, 34-40. [CrossRef]

4. Biesmans, G.; Mertens, A.; Duffours, L.; Woignier, T.; Phalippou, J. Polyurethane based organic aerogels and their transformation into carbon aerogels. J. Non-Cryst. Solids 1998, 225, 64-68. [CrossRef]

5. Rigacci, A.; Marechal, J.C.; Repoux, M.; Moreno, M.; Achard, P.J. Preparation of polyurethane-based aerogels and xerogels for thermal superinsulation. J. Non-Cryst. Solids 2004, 350, 372-378. [CrossRef]

6. Quignard, F.; Valentin, R.; Di Renzo, F. Aerogel materials from marine polysaccharides. New J. Chem. 2008, 32, 1300-1310. [CrossRef]

7. Cardea, S.; Gugliuzza, A.; Sessa, M.; Aceto, M.C.; Drioli, E.; Reverchon, E. Supercritical Gel Drying: A Powerful Tool for Tailoring Symmetric Porous PVDF-HFP Membranes. ACS Appl. Mater. Interfaces 2009, 1, 171-180. [CrossRef]

8. Zhang, X.; Chang, D.; Liu, J.; Luo, Y. Conducting polymer aerogels from supercritical $\mathrm{CO}_{2}$ drying PEDOT-PSS hydrogels. J. Mater. Chem. 2010, 20, 5080-5085. [CrossRef]

9. Korhonen, J.T.; Kettunen, M.; Ras, R.H.A.; Ikkala, O. Hydrophobic Nanocellulose Aerogels as Floating, Sustainable, Reusable, and Recyclable Oil Absorbents. ACS Appl. Mater. Interfaces 2011, 3, 1813-1816. [CrossRef]

10. Salerno, A.; Domingo, C. Making microporous nanometre-scale fibrous PLA aerogels with clean and reliable supercritical $\mathrm{CO}_{2}$ based approaches. Microporous Mesoporous Mater. 2014, 184, 162-168. [CrossRef]

11. Del Gaudio, P.; Auriemma, G.; Mencherini, T.; Porta, G.D.; Reverchon, E.; Aquino, R.P. Design of Alginate-Based Aerogel for Nonsteroidal Anti-Inflammatory Drugs Controlled Delivery Systems Using Prilling and Supercritical-Assisted Drying. J. Pharm. Sci. 2013, 102, 185-194. [CrossRef] [PubMed]

12. Meador, M.A.B.; McMillon, E.; Sandberg, A.; Barrios, E.; Wilmoth, N.G.; Mueller, C.H.; Miranda, F.A. Dielectric and Other Properties of Polyimide Aerogels Containing Fluorinated Blocks. ACS Appl. Mater. Interfaces 2014, 6, 6062-6068. [CrossRef] [PubMed] 
13. Randall, J.P.; Meador, M.A.B.; Jana, S.C. Tailoring Mechanical Properties of Aerogels for Aerospace Applications. ACS Appl. Mater. Interfaces 2011, 3, 613-626. [CrossRef] [PubMed]

14. Wang, X.; Jana, S.C. Synergistic Hybrid Organic-Inorganic Aerogels. ACS Appl. Mater. Interfaces 2013, 5, 6423-6429. [CrossRef]

15. Chen, H.-B.; Hollinger, E.; Wang, Y.-Z.; Schiraldi, D.A. Facile fabrication of poly(vinyl alcohol) gels and derivative aerogels. Polymer 2014, 55, 380-384. [CrossRef]

16. Schiraldi, D.A. Polymer Aerogels. In Encyclopedia of Polymer Science and Technology; John and Wiley and Sons: Hoboken, NJ, USA, 2015; pp. 1-18.

17. Leonhardt, E.E.; Meador, M.A.B.; Wooley, K.L. $\beta$-Cyclodextrin-Derived Monolithic, Hierarchically Porous Polyimides Designed for Versatile Molecular Separation Applications. Chem. Mater. 2018, 30, 6226-6230. [CrossRef]

18. Baldino, L.; Cardea, S.; Reverchon, E. Nanostructured chitosan-gelatin hybrid aerogels produced by supercritical gel drying. Polym. Eng. Sci. 2018, 58, 1494-1499. [CrossRef]

19. Li, M.; Qin, Z.; Cui, Y.; Yang, C.; Deng, C.; Wang, Y.; Kang, J.S.; Xia, H.; Hu, Y. Ultralight and Flexible Monolithic Polymer Aerogel with Extraordinary Thermal Insulation by A Facile Ambient Process. Adv. Mater. Interfaces 2019, 6, 1900314. [CrossRef]

20. Baldino, L.; Zuppolini, S.; Cardea, S.; Diodato, L.; Borriello, A.; Reverchon, E.; Nicolais, L. Production of biodegradable superabsorbent aerogels using a supercritical $\mathrm{CO}_{2}$ assisted drying. J. Supercrit. Fluids 2020, 156, 104681. [CrossRef]

21. Hu, J.; Zhu, J.; Ge, S.; Jiang, C.; Guo, T.; Peng, T.; Huang, T.; Xie, L. Biocompatible, hydrophobic and resilience graphene/chitosan composite aerogel for efficient oil-water separation. Surf. Coat. Technol. 2020, 385, 125361. [CrossRef]

22. Nita, L.E.; Ghilan, A.; Rusu, A.G.; Neamtu, I.; Chiriac, A.P. New Trends in Bio-Based Aerogels. Pharmaceutics 2020, 12, 449. [CrossRef] [PubMed]

23. Phadtare, V.D.; Parale, V.G.; Lee, K.-Y.; Kim, T.; Puri, V.R.; Park, H.-H. Flexible and lightweight Fe ${ }_{3} \mathrm{O}_{4} /$ polymer foam composites for microwave-absorption applications. J. Alloy. Compaunds 2019, 805, 120-129. [CrossRef]

24. Phadtare, V.D.; Parale, V.G.; Kim, T.; Lee, K.-Y.; Pathak, S.; Park, H.-H. Facile synthesis of a lightweight three-dimensional polymer scaffold dip-coated with multiple layers of $\mathrm{TiO}_{2}$ aerogel for X-band microwave absorption applications. J. Alloys Compd. 2020, 823, 153847. [CrossRef]

25. Daniel, C.; Longo, S.; Ricciardi, R.; Reverchon, E.; Guerra, G. Monolithic Nanoporous Crystalline Aerogels. Macromol. Rapid Commun. 2013, 34, 1194-1207. [CrossRef] [PubMed]

26. Venditto, V.; Pellegrino, M.; Califano, R.; Guerra, G.; Daniel, C.; Ambrosio, L.; Borriello, A. Monolithic polymeric aerogels with VOCs sorbent nanoporous crystalline and water sorbent amorphous phases. ACS Appl. Mater. Interfaces 2015, 7, 1318-1326. [CrossRef] [PubMed]

27. Daniel, C.; Pellegrino, M.; Venditto, V.; Aurucci, S.; Guerra, G. Nanoporous-crystalline poly(2,6-dimethyl-1,4phenylene)oxide (PPO) aerogels. Polymer 2016, 105, 96-103. [CrossRef]

28. Manfredi, C.; Del Nobile, M.A.; Mensitieri, G.; Guerra, G.; Rapacciuolo, M. Vapor sorption in emptied clathrate samples of syndiotactic polystyrene. J. Polym. Sci. Part B Polym. Phys. 1997, 35, 133-140. [CrossRef]

29. Mahesh, K.P.O.; Sivakumar, M.; Yamamoto, Y.; Tsujita, Y.; Yoshimizu, H.; Okamoto, S. Structure and properties of the mesophase of syndiotactic polystyrene: IX. Preferential sorption behavior of sPS-p-chlorotoluene mesophase membrane in a mixture of solvents. J. Membr. Sci. 2005, 262, 11-19. [CrossRef]

30. Daniel, C.; Longo, S.; Fasano, G.; Vitillo, J.G.; Guerra, G. Nanoporous Crystalline Phases of Poly(2,6-Dimethyl1,4-phenylene)oxide. Chem. Mater. 2011, 23, 3195-3200. [CrossRef]

31. Daniel, C.; Zhovner, D.; Guerra, G. Thermal Stability of Nanoporous Crystalline and Amorphous Phases of Poly(2,6-dimethyl-1,4-phenylene) Oxide. Macromolecules 2013, 46, 449-454. [CrossRef]

32. Daniel, C.; Antico, P.; Yamaguchi, H.; Kogure, M.; Guerra, G. Microporous-crystalline Microfibers by Eco-friendly Guests: An Efficient Tool for Sorption of Volatile Organic Pollutants. Microporous Mesoporous Mater. 2016, 232, 205-210. [CrossRef]

33. Daniel, C.; Antico, P.; Guerra, G. Etched Fibers of Syndiotactic Polystyrene with Nanoporous-Crystalline Phases. Macromolecules 2018, 51, 6138-6148. [CrossRef]

34. Joseph, A.M.; Nagendra, B.; Shaiju, P.; Surendran, K.P.; Gowd, E.B. Aerogels of Hierarchically Porous Syndiotactic Polystyrene with a Dielectric Constant Near to Air. J. Mater. Chem. C 2018, 6, 360-368. [CrossRef] 
35. Kim, S.J.; Chase, G.; Jana, S.C. Polymer aerogels for efficient removal of airborne nanoparticles. Sep. Purif. Technol. 2015, 156, 803-808. [CrossRef]

36. Kim, S.J.; Chase, G.; Jana, S.C. The role of mesopores in achieving high efficiency airborne nanoparticle filtration using aerogel monoliths. Sep. Purif. Technol. 2016, 166, 48-54. [CrossRef]

37. Alegre, C.; Sebastián, D.; Baquedano, E.; Gálvez, M.E.; Moliner, R.; Lázaro, M.J. Tailoring Synthesis Conditions of Carbon Xerogels towards Their Utilization as Pt-Catalyst Supports for Oxygen Reduction Reaction (ORR). Catalysts 2012, 2, 466-489. [CrossRef]

38. Sacco, O.; Vaiano, V.; Daniel, C.; Navarra, W.; Venditto, V. Removal of Phenol in Aqueous Media by N-doped $\mathrm{TiO}_{2}$ Based Photocatalytic Aerogels. Mater. Sci. Semicond. Process. 2018, 80, 104-110. [CrossRef]

39. Wan, W.; Zhang, R.; Mab, M.; Zhou, Y. Monolithic Aerogel Photocatalysts: A Review. J. Mater. Chem. A 2018, 6, 754-775. [CrossRef]

40. Maleki, H.; Hüsing, N. Current status, opportunities and challenges in catalytic and photocatalytic applications of aerogels: Environmental protection aspects. Appl. Catal. B Environ. 2018, 221, 530-555. [CrossRef]

41. Sacco, O.; Vaiano, V.; Daniel, C.; Navarra, W.; Venditto, V. Highly Robust and Selective System for Water Pollutants Removal: How to Transform a Traditional Photocatalyst into a Highly Robust and Selective System for Water Pollutants Removal. Nanomaterials 2019, 9, 1509. [CrossRef]

42. Dreyer, D.R.; Jia, H.-P.; Bielawski, C.W. Graphene Oxide: A Convenient Carbocatalyst for Facilitating Oxidation and Hydration Reactions. Angew. Chem. Int. Ed. 2010, 49, 6813-6816. [CrossRef]

43. Navalon, S.; Dhakshinamoorthy, A.; Alvaro, M.; Garcia, H. Carbocatalysis by Graphene-Based Materials. Chem. Rev. 2014, 114, 6179-6212. [CrossRef] [PubMed]

44. Peng, L.; Zheng, Y.; Li, J.; Jin, Y.; Gao, C. Monolithic Neat Graphene Oxide Aerogel for Efficient Catalysis of $\mathrm{S} \rightarrow \mathrm{O}$ Acetyl Migration. ACS Catal. 2015, 5, 3387-3392. [CrossRef]

45. Wen, G.; Wu, S.; Li, B.; Dai, C.; Su, D.S. Active Sites and Mechanisms for Direct Oxidation of Benzene to Phenol over Carbon Catalysts. Angew. Chem. Int. Ed. 2015, 54, 4105-4509. [CrossRef] [PubMed]

46. Dreyer, D.R.; Bielawski, C.W. Carbocatalysis: Heterogeneous carbons finding utility in synthetic chemistry. Chem. Sci. 2011, 2, 1233-1240. [CrossRef]

47. Acocella, M.R.; Mauro, M.; Guerra, G. Regio- and Enantioselective Friedel-Crafts Reactions of Indoles to Epoxides Catalyzed by Graphene Oxide: A Green Approach. ChemSusChem 2014, 7, 3279-3283. [CrossRef]

48. Acocella, M.R.; De Pascale, M.; Maggio, M.; Guerra, G. Graphite oxide as catalyst for diastereoselective Mukaiyama aldol reaction of 2-(trimethylsilyloxy)furan in solvent free conditions. J. Mol. Catal. A Chem. 2015, 408, 237-241. [CrossRef]

49. Acocella, M.R.; Maggio, M.; Ambrosio, C.; Aprea, N.; Guerra, G. Oxidized Carbon Black as an Activator of Transesterification Reactions under Solvent-Free Conditions. ACS Omega 2017, 2, 7862-7867. [CrossRef]

50. Upadhyay, R.K.; Soin, N.; Roy, S.S. Role of graphene/metal oxide composites as photocatalysts, adsorbents and disinfectants in water treatment: A review. RSC Adv. 2014, 4, 3823-3851. [CrossRef]

51. Kim, T.; Parale, V.G.; Jung, H.-N.-R.; Kim, Y.; Driss, Z.; Driss, D.; Bouabidi, A.; Euchy, S.; Park, H.-H. Facile Synthesis of $\mathrm{SnO}_{2}$ Aerogel/Reduced Graphene Oxide Nanocomposites via in Situ Annealing for the Photocatalytic Degradation of Methyl Orange. Nanomaterials 2019, 9, 358. [CrossRef]

52. Xiang, C.; Cox, P.J.; Kukovecz, A.; Genorio, B.; Hashim, D.P.; Yan, Z.; Peng, Z.; Hwang, C.-C.; Ruan, G.; Samuel, E.L.G.; et al. Functionalized Low Defect Graphene Nanoribbons and Polyurethane Composite Film for Improved Gas Barrier and Mechanical Performances. ACS Nano 2013, 7, 10380-10386. [CrossRef] [PubMed]

53. Nassira, H.; Sánchez-Ferrer, A.; Adamcik, J.; Handschin, S.; Mahdavi, H.; Taheri Qazvini, N.; Mezzenga, R. Gelatin-Graphene Nanocomposites with Ultralow Electrical Percolation Threshold. Adv. Mater. 2016, 28, 6914-6920. [CrossRef] [PubMed]

54. Joseph, A.M.; Nagendra, B.; Surendran, K.P.; Gowd, E.B. Sustainable in Situ Approach to Covalently Functionalize Graphene Oxide with POSS Molecules Possessing Extremely Low Dielectric Behavior. Langmuir 2019, 35, 4672-4681. [CrossRef] [PubMed]

55. Longo, S.; Mauro, M.; Daniel, C.; Musto, P.; Guerra, G. Rayleigh scattering by graphene-oxide in syndiotactic polystyrene aerogels. Carbon 2014, 77, 896-905. [CrossRef]

56. De Rosa, C.; Guerra, G.; Petraccone, V.; Pirozzi, B. Crystal structure of the emptied clathrate form $\left(\delta_{\mathrm{e}}\right.$ form) of syndiotactic polystyrene. Macromolecules 1997, 30, 4147-4152. [CrossRef] 
57. Nagendra, B.; Cozzolino, A.; Daniel, C.; Rizzo, P.; Guerra, G.; Auriemma, F.; De Rosa, C.; D’Alterio, M.; Tarallo, O.; Nuzzo, A. Two nanoporous crystalline forms of poly (2,6-dimethyl-1,4-phenylene) oxide and related Co-crystalline forms. Macromolecules 2019, 52, 9646-9656. [CrossRef]

58. Torres, F.J.; Civalleri, B.; Meyer, A.; Musto, P.; Albunia, A.R.; Rizzo, P.; Guerra, G. Normal Vibrational Analysis of the Syndiotactic Polystyrene s(2/1)2 Helix. J. Phys. Chem. B 2009, 113, 5059-5071. [CrossRef]

59. Hummers, W.S.; Offeman, R.E. Preparation of Graphitic Oxide. J. Am. Chem. Soc. 1958, 80, 1339. [CrossRef]

60. Ko, E.I. Aerogels in Kirk-Othmer Encyclopedia of Chemical Technology, 4th ed.; John Wiley \& Sons: Hoboken, NJ, USA, 1998; pp. 1-22.

Sample Availability: Samples of the compounds are not available from the authors.

Publisher's Note: MDPI stays neutral with regard to jurisdictional claims in published maps and institutional affiliations.

(C) 2020 by the authors. Licensee MDPI, Basel, Switzerland. This article is an open access article distributed under the terms and conditions of the Creative Commons Attribution (CC BY) license (http://creativecommons.org/licenses/by/4.0/). 